Review

\title{
Combination Strategies Using EGFR-TKi in NSCLC Therapy: Learning from the Gap between Pre-Clinical Results and Clinical Outcomes
}

\author{
Zheng Yang, Kin Yip Tam ${ }^{\bowtie}$ \\ Faculty of Health Science, University of Macau, Taipa, Macau, China \\ $\triangle$ Corresponding author: Dr. Kin Yip TAM, Faculty of Health Science, University of Macau, Taipa, Macau, China. Tel: +853-88224988; Fax: +853-88222314; \\ E-mail: kintam@umac.mo. \\ (C) Ivyspring International Publisher. This is an open access article distributed under the terms of the Creative Commons Attribution (CC BY-NC) license \\ (https://creativecommons.org/licenses/by-nc/4.0/). See http://ivyspring.com/terms for full terms and conditions.
}

Received: 2017.09.23; Accepted: 2017.12.09; Published: 2018.02.05

\begin{abstract}
Although epidermal growth factor receptor (EGFR) inhibitors have been used to treat non-small cell lung cancer (NSCLC) for decades with great success in patients with EGFR mutations, acquired resistance inevitably occurs after long-term exposure. More recently, combination therapy has emerged as a promising strategy to overcome this issue. Several experiments have been carried out to evaluate the synergism of combination therapy both in vitro and in vivo. Additionally, many clinical studies have been carried out to investigate the feasibility of treatment with EGFR-tyrosine kinase inhibitors (TKi) combined with other NSCLC treatments, including radiotherapy, cytotoxic chemotherapies, targeted therapies, and emerging immunotherapies. However, a significant gap still exists when applying pre-clinical results to clinical scenarios, which hinders the development and use of these strategies. This article is a literature review analysing the rationalities and controversies in the transition from pre-clinical investigation to clinical practice associated with various combination strategies. It also highlights clues and challenges regarding future combination therapeutic options in NSCLC treatment.
\end{abstract}

Key words: Combination; EGFR-TKi; NSCLC; chemotherapy; targeted therapy; immunotherapy

\section{Introduction}

Cancer is still one of the leading causes of death from illness, both in developed and developing countries [1]. Approximately 14.1 million newly diagnosed cases and 8.2 million deaths have been reported throughout the world according to the data set GLOBOCAN, which includes data on the annual increase in social medical burden [2]. Among all cancer types, lung cancer causes the highest number of cancer-related deaths in males, and is among the top five causes of cancer-related deaths in females [3]. In total, 1.8 million new cases of lung cancer were diagnosed in 2015, with a half of them living in underdeveloped areas [4]. Of note, in contrast to the decreasing incidence in Western countries, lung cancer diagnoses and death in China are still rising [5], pointing to a tough and long road ahead to control the morbidity and mortality associated with lung cancer.

It has been reported that NSCLC contributes to over $80 \%$ of all lung cancer cases [6]. Platinum cytotoxic chemotherapy as well as radiotherapy emerged approximately half a century ago as therapeutic strategies for patients with NSCLC [7]. Although the application of those treatments with surgical operation was beneficial for some patients, by relieving pain, prolonging lifespan, and improving the quality of life [8], the occurrence of adverse effects that not only killed cancer cells but also damaged the normal tissues and organs hampered the extensive application of those strategies [9]. With the development of individualised treatment, it has become well appreciated that lung cancer patients 
with certain genetic backgrounds would benefit from targeted therapy [10]. EGFR is one of the members of the erbB family, a series of transmembrane growth factor receptor tyrosine kinase proteins, including erbB1 (EGFR), erbB2 (HER2), erbB3 (HER3), and erbB4 (HER4) [11]. Approximately 30\% to $40 \%$ of NSCLC patients harbour an EGFR mutation [6], which promotes the constant phosphorylation of EGF receptors, activates downstream signalling pathways such as RAS-RAF-MEK-ERK, PI3K-AKT-mTOR, and JAK-STAT, and in turn confers cancer cell proliferation and survival [12]. Therefore, targeting EGFR is a promising therapeutic strategy for NSCLC patients with an EGFR mutation, which have been highly successful in recent decades [13].

Unfortunately, acquired resistance to EGFR-TKi treatment, where lung cancer cells become insensitive to those inhibitors after a long period of treatment, almost inevitably occurs [14]. Possible mechanisms for the acquired resistance have been extensively discussed. Appearance of second-site hot spot mutations that partially contributed to the acquired resistance, such as T790M [15], D761Y [16], and T854A [17], with T790M being the most common alteration, led to the discovery of the third-generation EGFR-TKis, such as AZD9291 [18] and CO-1686 [19]. Other mechanisms leading to resistance not involving EGFR mutations included the bypass signalling activation such as the amplification of mesenchymal-epithelial transition (MET) [20], a type of human hepatocyte growth factor receptor that is essential for embryonic development, organogenesis, and wound healing, or a mutation in the PI3K pathway [21] found in some NSCLC patients who did or did not undergo EGFR inhibitor treatment. Occurrence of epithelial-mesenchymal transition (EMT) was reported to confer acquired resistance to EGFR targeted therapy [22]. Combination strategy has been suggested as a promising method not only to overcome resistance but also to reduce the dosages of both agents to minimise adverse effects and enhance the anti-cancer effect of individual strategies [23]. The current review summarises EGFR-TKi combinations for treating NSCLC, including radiotherapy, chemotherapy, targeted therapy, and emerging immunotherapy. The focus is on both clinical outcomes and pre-clinical evaluations, to analyse the pros and cons of various combination strategies and to elucidate clues for future therapeutic options in clinical practice.

\section{EGFR-TKi combined with radiotherapy}

Radiotherapy is widely used in the treatment of NSCLC [24]. It is usually used as an adjuvant treatment postoperatively. However, for patients with locally advanced NSCLC who cannot undergo surgery, radiotherapy combined with chemotherapy is helpful [24]. The rationale of administering EGFR-TKi with radiotherapy is based on the involvement of EGFR overexpression or mutation in radio-resistant cases of NSCLC. Chinnaiyan et al. [25] reported the synergistic anti-cancer effect of erlotinib combined with radiotherapy in UM-SCC6 and H226 human NSCLC cell lines via attenuating cell clonogenic survival and in vivo tumour growth. These effects appeared to be attributed to the addition of erlotinib to radiation therapy, which induced cell apoptosis, inhibited EGFR activation, overexpressed Rad51, and enhanced other signalling mediators, such as Egr-1, IL-1 $\beta$, and CXCL1 [25]. Kim et al. [26] found EGFR expression in three different NSCLC cell lines to be positively correlated with sensitivity to EGFR-TKi treatment, but to be negatively correlated to radio-sensitivity. They found that the involvement of erlotinib could not only increase G1 cell cycle arrest in those cell lines, but also promote cell apoptosis in A431 [26]. Michael et al. [27] summarised several pre-clinical studies and addressed various combination mechanisms apart from above-mentioned ones, including inactivating cancer stem cells directly to improve local tumour control, modulating several signalling pathways, suppressing DNA repair, decreasing cell repopulation, and improving re-oxygenation, all of which provided valid evidences for clinical practice. Several trials have tested the combination of EGFR-TKi and radiotherapy, and some have reported that the combination would be feasible, tolerated, and effective in certain populations of patients [28], indicating that the use of the combination should be determined individually for specific cases based on the patient's genetic background.

Besides chest irradiation, local radiotherapy limited to the brain region is a treatment option for NSCLC patients with brain metastasis, which includes $20 \%$ to $40 \%$ of all NSCLC cases [29]. These patients account for a large proportion of NSCLC-associated deaths. The brain-blood barrier (BBB), which restricts the ability of systematic chemotherapeutic agents and EGFR-TKi to reach the cerebrospinal fluid (CSF) [30], can be damaged when brain metastasis occurs. The resultant leakage of the BBB in turn improves the penetration ability of these agents or inhibitors, which could be applied together with whole-brain radiotherapy (WBRT) [31]. Many clinical trials have reported the availability of WBRT combined with EGFR-TKi for patients with brain metastasis. Cai et al. [32] found that, compared with radiotherapy alone, the addition of EGFR-TKi significantly increased patients' progression-free 
survival (PFS) and overall survival (OS). Fan et al. [33] showed a significant difference in median survival time (MST) between cases with an EGFR mutation and those with wild-type EGFR (22.0 vs 7.5 months, $p=0.0001)$. Lu et al. [34] reported the significant improvement in OS as the combination controlled metastatic intracranial lesions. However, controversy remains over whether the combination can bring about realistic benefits to patients, including limited improvement in OS compared with WBRT alone [35, 36], or severe toxicity up to over grade 3 [37]. These results suggested that the application of these combination should be carefully weighed for NSCLC patients with brain metastasis, until significant improvement of OS outweighs the potential adverse effects, or until those adverse effects become manageable. It is worth mentioning that, in recent years, osimertinib, the next-generation EGFR-TKi, was proved to have better penetration through $\mathrm{BBB}$ than gefitinib, which led to the enhanced activity in leptomeningeal disease therapy [38]. In comparison with platinum-pemetrexed chemotherapy, administration of osimertinib showed significantly higher ORR and longer PFS in advanced NSCLC patients with CNS metastasis [39]. These results indicated that osimertinib could be one of the promising strategies for NSCLC patients with brain metastasis that may outperform radiation and combination therapy.

\section{EGFR-TKi in combination with chemotherapy}

In the past, patients who suffered from various types of cancer significantly benefited from classical cytotoxic drug chemotherapy, administered either simultaneously with surgery to increase the success of the operation or continuously in unresectable cases to control the progression of diseases and improve the patient's quality of life [8]. Chemotherapy is still recommended as the first-line therapy in NSCLC patients without defined therapeutic targets [40], although several treatments combining chemotherapy and EGFR-TKi are under development.

Platinum-based chemotherapy (cisplatin or carboplatin) is often used to treat NSCLC [7], whereas its combination with EGFR-TKi showed mixed results in both pre-clinical studies and in clinical trials. Lee $e t$ al. [41] indicated that a combination of low-dose erlotinib and cisplatin may exert a synergistic effect in NSCLC cell lines that contain an EGFR exon 19 deletion, which is probably due to an anti-angiogenesis effect via targeting c-myc/HIF-1a/VEGF signalling. However, a meta-analysis that included 191 randomised phase II trials concluded that the combination of erlotinib plus carboplatin and paclitaxel did not have a significant beneficial effect on PFS and OS but rather showed more severe adverse effects compared to erlotinib alone, regardless of the EGFR status [42]. Furthermore, Tsai et al. [43] reported that the failure of the TKi-platinum combination was probably due to the antagonistic effect of the two compounds, as EGFR-TKi can interfere with the absorption of cisplatin. Liu et al. [44] demonstrated that the inhibition of autophagy could improve the synergism of the gefitinib-cisplatin combination. Future studies will probably reveal the reason for the failure to translate these "promising" pre-clinical results into clinical effects, and provide possible solutions that will allow these combination therapies to be used in clinical practice (Figure. 1).

Gemcitabine is another cytotoxic agent used in NSCLC therapy. It has been reported that the combination of gemcitabine and AG1478, an EGFR-TKi, produces a synergistic anti-cancer effect depending on the level of ERK activation as well as KRAS status [45]. Nonetheless, a multicentre, randomised, open-label phase trial showed that sequentially delivered erlotinib and gemcitabine did not provide beneficial effects to patients over gemcitabine alone [46]. A recent systematic analysis summarised a number of clinical trials of combination therapy using EGFR-TKi together with various cytotoxic chemotherapeutic agents and concluded that the combination is not superior to either agent alone for the first-line treatment of NSCLC [47]. Thus, further studies are required to determine the possible effectiveness and mechanisms of combination therapy.

Pemetrexed has recently been developed to suppress the metabolism of intracellular folic acid, and thus prevent cell division [48]. It has significant activity and favourable tolerance as well as low toxicity when compared to other cytotoxic agents, and has been well studied for administration either alone or in combination [48]. A combination of pemetrexed and EGFR-TKi has been highly touted to overcome EGFR-TKi-acquired resistance. In their study, $\mathrm{Wu}$ et al. [49] illustrated that the synergistic anti-cancer effect of the combination can only be found in the EGFR-TKi resistant PC9/GR cell line and not in the EGFR-TKi sensitive PC9 cell line, with a possible mechanism of inducing cell apoptosis and suppressing AKT as well as ERK phosphorylation in synergy. La Monica et al. [50] demonstrated that the sequence of EGFR-TKi and pemetrexed administration is crucial for EGFR-TKi-resistant cell lines to respond well to the treatment. Clinical trials have confirmed the synergism of the combination, and that the synergism is dependent on EGFR genetic 
status instead of the presence of resistance. In NSCLC patients with an EGFR-sensitive mutation, the combination produced better PFS or objective response rate (ORR) with acceptable toxicity compared to those produced by either EGFR-TKi or pemetrexed alone [51-53]. However, in patients with wild-type EGFR, studies on the combination were reported to be either invalid [54] or terminated without published results [55]. Further clinical studies are required to determine of the most suitable cases for pemetrexed-EGFR-TKi combination treatment (Figure. 1).

\section{Pre-Clinically}

Clinically

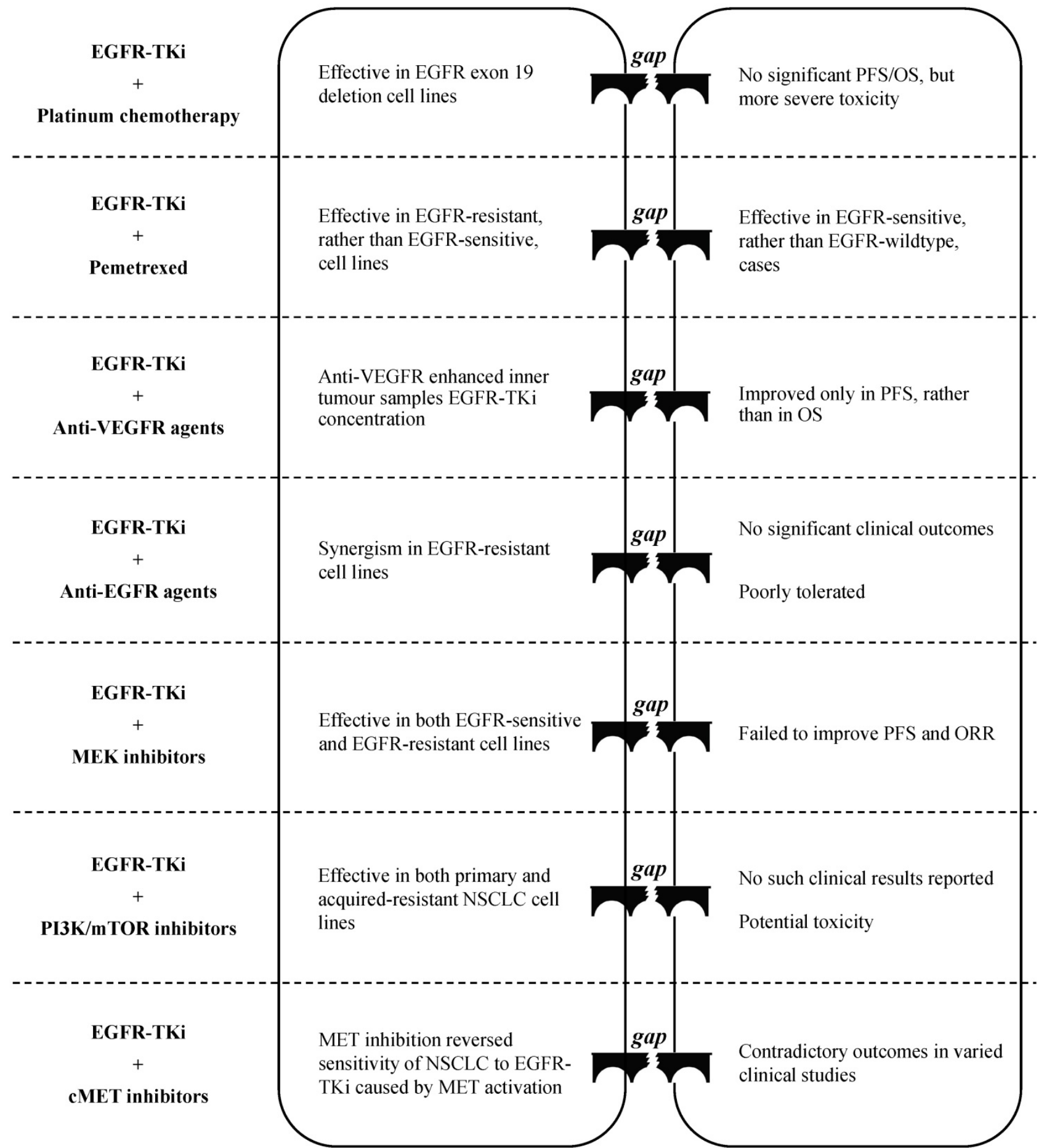

Figure. 1 Gaps between pre-clinical evaluation and clinical outcome when EGFR-TKi combined with chemotherapy/targeted therapy 


\section{EGFR-TKi combined with targeted therapy}

Although classical cytotoxic chemotherapy has been widely used for decades, the relief of cancer burden is usually accompanied with significant adverse effects, such as myelosuppression, in most cases [56]. Therefore, targeted therapy was developed to treat the disease with fewer side effects. This approach has achieved tremendous success in the last two decades in specific groups of patients, holding the promise of emerging personalised medicine [57]. A large number of inhibitors have been discovered for targeting specific signaling pathways, including VEGFR/angiogenesis [56], EGFR/RAS/RAF/MEK/ ERK [57], PI3K/AKT/mTOR [58], and JAK/STAT [59]. Studies of these targeted therapies as part of combination treatment are still underway in both pre-clinical and clinical studies.

\section{Anti-VEGFR2 agents}

Vascular endothelial growth factor receptor 2 (VEGFR2), one of the subunits of the VEGFR family, is strongly associated with angiogenesis which plays a key role in tumour progression and metastasis [60]. As micro-vessel count was reported to be an independent prognostic predictor of NSCLC [61], targeting VEGFR2 should be a promising strategy for NSCLC treatment. Bevacizumab is a promising antibody for blocking VEGFR2, and is used in combination with EGFR-TKi as the first-line treatment for NSCLC [62]. Li et al. [63] found that the addition of bevacizumab to EGFR-TKi in NSCLC xenografts enhanced the TKi concentration in tumour samples with high levels of VEGF protein, the direct ligand of VEGFR2, suggesting that the combination of targeting EGFR and VEGFR2 may produce a synergistic anti-cancer effect. Clinical data have shown that the combination may be significantly beneficial for patients with specific genetic backgrounds. An open-label, randomised, multicentre, phase II study showed a significant extension of PFS in patients with EGFR-mutation positive when treated with erlotinib plus bevacizumab compared with erlotinib alone [64]. Although the results of another phase II trial failed to meet its primary goal, it showed that the combination of gefitinib and bevacizumab resulted in longer PFS in the sub-group of patients with an EGFR-sensitive mutation of exon 19 deletion [65]. A recent meta-analysis showed that patients with T790M-positive tumours may have a significant improvement in PFS compared to that of patients with T790M-negative tumours [66]. Several clinical trials are in progress, either in the first-line [67] or the second-line therapy [68], to evaluate the feasibility of an EGFR-TKi and bevacizumab combination as well as to determine eligible patients for this therapy.

Small molecules that target VEGFR2 have also been discovered. However, none of them are VEGFR2-specific inhibitors. Vandetanib, developed by AstraZeneca as a VEGFR and EGFR dual inhibitor, has been included in several clinical trials from phase I to phase III. It has shown a favourable effect and acceptable toxicity [69]. BMS-690514, also a dual inhibitor that targets both VEGFR and EGFR that showed a significant anti-tumour effect in NSCLC xenografts [70], was evaluated in a phase I/IIa clinical trial. It was found to have anti-tumour activity and manageable toxicity in patients with NSCLC regardless of EGFR status [71]. Cabozantinib, an oral small-molecule inhibitor of VEGFR2 and MET, showed a significant increase in PFS and OS compared to erlotinib, but showed no further improvement when erlotinib was added as a combination [72]. As most of these trials are based on the second-line or the third-line NSCLC therapy [69, 72], these novel VEGFR small molecules can be developed as an option for patients who have failed to respond to previous chemotherapies or targeted therapies.

\section{Anti-erbB family agents}

EGFR, one of the sub-receptors in the erbB family (erbB1), is composed of an extracellular domain, a transmembrane domain, and an intracellular kinase domain [73]. EGFR-TKis bind the intracellular domain of EGFR to suppress erbB dimerisation, which in turn blocks downstream signal transduction [74]. Numerous anti-EGFR monoclonal antibodies have been developed to target the extracellular domain of EGFR to prevent its ligand from activating the receptor [75]. Cavazzoni et al. [76] studied the combination of cetuximab, the first developed anti-EGFR monoclonal antibody, and erlotinib, and found that the application of erlotinib could induce the accumulation of EGFR protein via protein stabilisation and could increase the binding sites of NSCLC tumour cells to cetuximab, which potentiated the cetuximab-dependent cell cytotoxicity and enabled a synergistic anti-cancer effect in vitro and in vivo. Wang et al. [77] reported that in NSCLC cells harbouring an EGFR-T790M-resistant mutation, combined erlotinib and cetuximab led to significant cell growth inhibition, induction of apoptosis, and EGFR signal suppression. These results indicate a probable synergism between these two anti-EGFR compounds. Although several phase I clinical trials $[78,79]$ have shown good tolerance to the combination of EGFR-TKi and anti-EGFR antibodies and have suggested the need for further studies on the 
efficacy of the combination, phase II/III studies with published results are rare except for several case reports on the feasibility of the afatinib and cetuximab combination [80, 81]. Moreover, administration of cetuximab after treatment with a TKi showed no significant clinical response [82].

Overexpression of HER2, another member of the erbB family, is also found in various types of cancer including NSCLC, and is associated with a poor clinical outcome [83]. Pre-clinical studies have found that the amplification of HER2 in NSCLC enhanced sensitivity to treatment with gefitinib via the inhibition of HER2/HER3 heterodimer formation [84], implying the possibility of combination therapy with the addition of an anti-HER2 antibody to EGFR-TKi treatment. Nakamura et al. [85] found that the combination of gefitinib and trastuzumab, the well-known anti-HER2 antibody, exerted a synergistic effect on cell proliferation attenuation through co-suppression of EGFR downstream signalling in the NSCLC cell line A549, which is driven by EGFR/HER2 heterodimers, but not in NCI-H23, which is controlled by other factors or receptors. Friess et al. [86] demonstrated the synergistic anti-tumour effects of a combination of erlotinib and pertuzumab, another marketed anti-HER2 antibody, in three NSCLC xenograft models regardless of HER2 expression levels. However, positive outcomes after treatment with these combinations have been sparsely reported in clinical practice. Unfortunately, it has been reported that the combination of pertuzumab and erlotinib is poorly tolerated in patients with relapsed NSCLC [87]. The results of these summarised clinical trials with the application of anti-HER2 therapy in NSCLC cases, either as monotherapy or in combination, are controversial [88], indicating that more studies are required to determine the exact role of anti-HER2 treatment in NSCLC before further clinical application.

\section{Anti-EGFR downstream agents}

The disruption in EGFR downstream signalling has also been shown to contribute to the acquired resistance of NSCLC to EGFR-TKi therapy [89, 90]. One such signalling pathway is RAS-RAF-MEK-MAPK, the inhibition of which would be a promising strategy to tackle acquired resistance in NSCLC patients who have previously undergone EGFR-TKi treatment. Sorafenib, a multi-target anti-cancer drug used to treat liver cancer, can suppress the activation of MEK as well as its downstream target, MAPK, which can in turn inhibit cell and tumour growth in various EGFR-TKi-resistant lung cancer cell lines and in vivo [91]. Balko et al. [92] found that inhibition ERK activity, which is essential for NSCLC cell proliferation, by applying its up-stream MEK1/2 inhibitor U0126, can reduce NSCLC cell survival, induce cell apoptosis and sensitise those cell lines to the treatment with EGFR inhibitors. Song et al. [93] reported that the application of either CZ0775 or AZD6244, two MEK1/2 dual inhibitors, along with the EGFR inhibitor lapatinib showed a synergistic anti-cancer effect in both EGFR-sensitive and EGFR-resistant NSCLC cell lines via the promotion of caspase-3 dependent cell apoptosis. Li et al. [94] also confirmed the synergism of AZD6244 with gefitinib in promoting cell death in EGFR-TKi resistant NSCLC cell lines. Since two MEK inhibitors, cobimetinib and selumetinib, were approved by the Food and Drug Administration (FDA) for the treatment of melanoma [95, 96], several clinical trials have included those MEK inhibitors in combination with EGFR-TKis to investigate their combined effect in treating NSCLC $[97,98]$. However, recently published data indicated that the combination of AZD6244 with erlotinib failed to show improvement in ORR or PFS in comparison with monotherapy in both KRAS mutant and wild-type advanced NSCLC cases [99]. Moreover, the clinical outcomes in a phase III trial with the addition of AZD6244 to chemotherapy were not superior to those of chemotherapy alone [100]. These results suggest that the clinical application of MEK inhibitors for NSCLC therapy, either as monotherapy or in combination, should be further investigated.

PI3K-AKT-mTOR is another EGFR-controlled signalling pathway, in which the mutation or overexpression of a PI3K family protein continuously activates its downstream target [101]. It accounts for not only the formation and progression of many tumours but also the resistance to targeted inhibitors such as EGFR-TKi [102]. PTEN, a known tumour suppressor gene, that negatively regulates activation of AKT driven by PI3K [102], was found to be associated with elevated AKT phosphorylation in patients resistant to gefitinib treatment [103]. In contrast, the significantly higher expression of BIM and mTOR, two downstream factors in the PI3K pathway, was shown to be correlated with poor clinical outcomes in both OS and PFS in patients treated with erlotinib [104]. This result indicated that the abnormality in PI3K signalling contributes, at least in some NSCLC cases, to EGFR-TKi resistance. Therefore, several pre-clinical combination studies were carried out, which have shown promising results. For example, in one study, OSU-03012, which targets both ER stress and AKT, helped in overcoming the resistance of NSCLC cell lines to either erlotinib or gefitinib treatment [105]. The combination of everolimus, an mTOR inhibitor, and gefitinib showed 
synergism in both primary and resistance-acquired NSCLC cell lines in a dose-dependent manner [106]. However, it is uncommon to find these combinations in clinical trials [107-109]. In fact, marketed $\mathrm{PI} 3 \mathrm{~K} / \mathrm{AKT} / \mathrm{mTOR}$ inhibitors are rare, with just a few that are aimed at specific (advanced/refractory) cancer types [110-112]. Moreover, the potential adverse effects, including the suppression of myelocytes and immunocytes, toxicity to the liver and kidneys, and haematotoxicity [113], restrict the late-stage application of inhibitors as well as their potential combination with other TKis.

\section{MET inhibitors}

MET receptors, which transduce several downstream signalling targets that mediates the process of cell survival and proliferation, including MAPK, PI3K, STAT, and NF-kB, have been widely discussed as potential targets in cancer therapy [114]. Puri et al. [115] found that stimulation of EGF and HGF simultaneously in NSCLC cell lines promoted tumour cell growth and downstream signalling activation. Zhang et al. [116] reported that the overexpression of MET led to increased EGFR phosphorylation via EGFR heterodimerisation, whereas suppression of both MET and EGFR produced synergistic anti-cancer effects both in vitro and in vivo by inhibiting downstream signalling. These results point to a promising approach for treating NSCLC via targeting both MET and EGFR. Several pre-clinical studies have reported the feasibility of the combination in treating NSCLC. For example, the combination of gefitinib with either a HGF neutralising antibody or a MET inhibitor showed additive anti-cancer effects in transgenic mice with lung tumourigenesis [117]. Additionally, the application of the MET inhibitor INC-280 restored the sensitivity of NSCLC cell lines to erlotinib in the presence of HGF stimulation via increased cell apoptosis [118], and the addition of MET inhibitor tepotinib reversed resistance to EGFR inhibitors in NSCLC models mediated by MET activation [119]. However, results of clinical trials have been diversing, with several studies reaching their end points [120-122], whereas others have failed to fulfill the expected outcomes [123, 124]. Besides, amplified toxicity, witnessed as decreased maximum tolerated dose of the combination (crizotinib plus erlotinib) in comparison with either agents in monotherapy on the approved dose [125], or increased incidence of interstitial lung disease when additional tivantinib involved in erlotinib treated advanced NSCLC cases [126], resulted in termination of such clinical trials as well. In summary, KRAS mutations [123], strong MET amplification [121, 127], or a high level of extracellular
HGF [128] could be useful biomarkers in NSCLC patients, indicative of a good response to this combination, although further investigation in clinical trials is required. What is noteworthy is that the amplification of MET has been reported as an important mechanism in NSCLC patients resistant to treatment with osimertinib [129], a novel third-generation EGFR inhibitor [18], suggesting a potential application of combination therapy with compounds that lead to MET inhibition.

The effectiveness of targeted therapy involving EGFR-TKi combinations in pre-clinical studies is based on specific genetic alterations or on bypassing signal activation [130]. Nevertheless, the reasons for the failure of translating those strategies into clinical practice include, but are not limited to the following: the differences in the tumour microenvironment between humans and animals, or between in vivo and in vitro conditions [131], and the integrity of the tumour's internal environment, from which the tumour cannot be completely overcome via suppression of one or two individual genes or pathways [132]. The more we know about these differences, the more we will be able to make the right decisions and promote successful therapeutic effects for specific patients (Figure 1).

\section{EGFR-TKi combined with immunotherapy}

Recently, immunotherapy has become the most popular option for cancer treatment [133]. Unlike targeted therapy, which produces direct anti-tumour effects by blocking specific signalling pathways that are over-activated in cancer, the goal of immunotherapy is to stimulate in vivo immune cells to kill tumour cells that have escaped from previous immunological surveillance [134]. The antibodies used in immunotherapy are larger, which are highly specific and selectively bind their target receptors than those used in targeted therapy [135]. Therefore, they have potent anti-cancer effects. Application of large molecules for immunotherapy will become widely available, as many antibodies have been approved for NSCLC treatment [136, 137]; meanwhile, the combination of immunotherapy with targeted therapy is also under evaluation [138].

The programmed death 1 (PD-1) pathway and its key ligand, the programmed death ligand 1 (PD-L1) have emerged in recent years as key targets for NSCLC therapy. Several antibodies that target either PD-1 or PD-L1 have been developed and show encouraging clinical outcomes in patients with NSCLC [139]. Pre-clinical results have shown that EGF-stimulated EGFR activation upregulated PD-L1 expression, which induced T-cell apoptosis and 
contributed to the immune escape of both EGFR-sensitive and EGFR-resistant NSCLC cell lines [140]. D'Incecco et al. [141] reported that elevated PD-L1 levels are associated with the presence of EGFR mutations and, with EGFR-TKi treatment, higher ORR, time to progression (TTP), and OS. This suggests that the combination of anti-PD-1/PD-L1 and EGFR-TKi might have synergistic effects in NSCLC therapy. Although many clinical trials have attempted to study this combination in pre-treated NSCLC cases and have shown promising clinical activity, the much higher incidence of adverse effects, with most of them being grade $3 / 4$, impeded the progress of the clinical studies, and even led to termination [142]. Moreover, two key phase II/III clinical trials, namely Keynote-010 [143] and CheckMate-057 [144], respectively, have revealed that patients with EGFR-mutated NSCLC would have poorer survival outcomes by applying anti-PD-1 antibodies compared with those harboring EGFR-wild type. Considering the effectiveness of EGFR-TKi on EGFR-mutation positive, rather than negative cases, how to fully utilise the functional role of anti-PD-1/PD-L1 antibody for NSCLC therapy, especially for those cases with EGFR mutation, should be further evaluated.

Cytotoxic $\mathrm{T}$ lymphocyte-associated antigen- 4 (CTLA-4), another immune checkpoint receptor, has also been developed as a potential target for NSCLC therapy [145]. However, administration of anti-CTLA-4, a large molecule, even as monotherapy, frequently led to toxicity $[146,147]$. The safety issues encountered by multiple pipelines indicated that we lack sufficient knowledge about antibody-based immunotherapy, which should be overcome before the clinical application of immunotherapeutic drugs.

\section{Conclusions and Perspectives}

For patients with NSCLC who harbour EGFR mutations, marketed EGFR-TKis (Table 1) are the first-line strategy recommended in the NCCN guidelines [40]. It will remain the preferred option for a long period until a better choice emerges. Therefore, acquired resistance will be one of the most challenging issues to overcome. Even for the new-generation EGFR-TKis, they are not insusceptible to acquired resistance [149]. Mechanism of drug actions for various kinds of combination strategies have been reported in pre-clinical studies. As shown in Figure 2, these can be broadly summarised as: 1). Promoting cell apoptosis synergistically $[25,49,77,92$, 117]; 2). Co-suppression in EGFR and its downstream signalling activation $[25,49,77,85,92,105,140]$; 3). One drug facilitating the other to enhance drug sensitivity [63, 76]. However, it is rare that these pre-clinical reported "promising" combination strategies can be translated into clinical practice successfully, suggesting the mechanisms of drug actions discovered in pre-clinical studies are unlikely to pertain to the clinical situations. Clearly, biomedical scientists and physicians should work closely to rationalise the gaps between pre-clinical results and clinical outcomes. In the foreseeable future, radiotherapy and chemotherapy will still be used as adjuvant or neoadjuvant therapy to combine with EGFR-TKi in cases of advanced, refractory, or metastatic NSCLC as long as the effectiveness can be validated in clinical trials. The use of combined targeted therapy with EGFR-TKi should be case dependent, as diverse signalling activation will be developed differently in different patients. A comprehensive application of chip-based diagnose techniques, next-generation sequencing, and immunohistochemistry to monitor potential biomarkers in circulation or in tumour samples will be helpful to determine and provide appropriate treatment options for specific cases. For combination therapy involving immunotherapy, it is crucial to avoid the increased adverse effects caused by the administration of antibodies, which is a problem awaiting detailed mechanistic elucidation. Therefore, it is important to find out the sub-population with the best response to the combination therapy.

Moreover, development of alternative strategies should be encouraged, in addition to the previous options, to cure individual NSCLC cases. Targeting tumour metabolism is one of the emerging and promising strategies for cancer treatment recently, which refers to the therapeutic modulations of the specific process of energy metabolism that cancer cells adopted, namely metabolising glucose through the anaerobic glycolysis rather than the normal mitochondrial oxidative phosphorylation (OXPHOS) even in normoxia conditions [150]. Traditional chemotherapies and targeted therapies produce direct insult to cancer cells, such as cisplatin/carboplatin which destroy the function of DNA and produce cell cycle arrest [151], or target inhibitors that block specific signaling pathways leading to cancer cell death $[13,62,114]$. However, it is the dysfunction of cancer cell metabolism that conferred the Warburg Effect [152], while targeting this phenotype could be a strategy to expose tumour in an unfavourable micro-environment. This hypothesis has inspired many scientists to focus on several enzymes that would be crucial in the process of cell metabolism, such as glucose transporters (GLUTs) [153], hexokinase2 (HK2) [154], pyruvate kinase M2 (PKM2) [155], pyruvate dehydrogenase kinase (PDK) [156], and lactate dehydrogenase-A (LDHA) [157]. A 
number of inhibitors that target those specific enzymes have been developed as potential anti-cancer agents [158]. However, researches that directly engaged in those cell metabolic inhibitor in combination with EGFR-TKi are rare, only with two papers recently reported the anti-cancer synergy in NSCLC cell lines or cancer xenograft model via EGFR-TKis combined with dichloroacetate [159] or 2,2-dichloroacetophenone [160], both of which are PDK inhibitors. Further investigations will be required to understand the mechanism of drug actions, which could facilitate the development of this combination strategy to overcome the resistance to EGFR-TKi treatment.

Last but not the least, retrospective studies should be performed to analyse not only the activity or toxicity of the combination but also the categorisation of patient subgroups with the aim to select patients that are likely to be benefited from combination therapy in terms of cost-effectiveness, extended lifespan, and improved quality of life.

Table. 1 Marketed EGFR inhibitors approved for NSCLC treatment
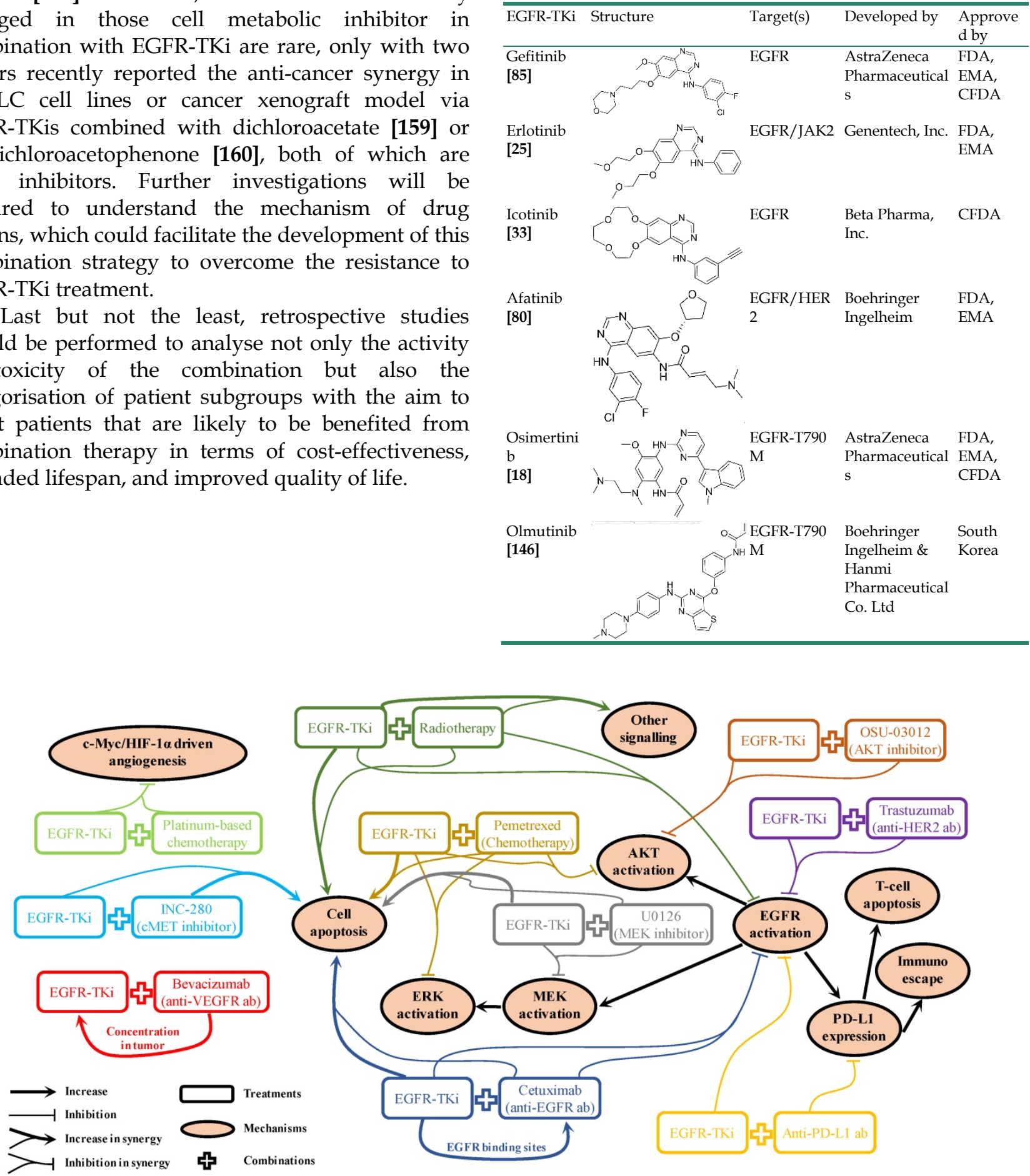

Figure. 2 Mechanism of drug actions for EGFR-TKi involved combination strategies reported in pre-clinical results

\section{Abbreviations}

EGFR, epidermal growth factor receptor; NSCLC, non-small cell lung cancer; TKi, tyrosine kinase inhibitors; MET, mesenchymal-epithelial transition; EMT, epithelial-mesenchymal transition;
BBB, brain-blood barrier; CSF, cerebrospinal fluid; WBRT, whole-brain radiotherapy; PFS, progression-free survival; OS, overall survival; MST, median survival time; VEGFR2, vascular endothelial growth factor receptor 2, OXPHOS, oxidative phosphorylation; GLUTs, glucose transporters; HK2, 
hexokinase2; PKM2, pyruvate kinase M2; PDK, pyruvate dehydrogenase kinase; LDHA, lactate dehydrogenase-A.

\section{Acknowledgements}

The authors thank Editage (online.editage.com) for English language editing. Thanks are due to Dr. Xiaohui $\mathrm{Hu}$ and Dr. Shaolin Zhang for helpful discussion. This work was supported by the Science and Technology Development Fund, Macao S.A.R (FDCT) (Project reference no. 086/2014/A2) and the University of Macau multiyear research grant (MYRG2016-00102-FHS).

\section{Competing Interests}

The authors have declared that no competing interest exists.

\section{References}

[1] Torre LA, Bray F, Siegel RL, et al. Global cancer statistics, 2012. CA Cancer J Clin. 2015; 65: 87-108.

[2] Ferlay J, Soerjomataram I, Dikshit R, et al. Cancer incidence and mortality worldwide: sources, methods and major patterns in GLOBOCAN 2012. Int J Cancer. 2015; 136 (5): e359-e386.

[3] Jemal A, Bray F, Center MM, et al. Global cancer statistics. CA Cancer J Clin. 2011; 61: 69-90.

[4] Chen W, Zheng R, Zeng H, et al. Epidemiology of lung cancer in China. Thorac Cancer. 2015; 6: 209-215.

[5] Silva MB, Almeida MA, Panato BP, et al. Clinical applicability of nursing outcomes in the evolution of orthopedic patients with Impaired Physical Mobility. Rev Lat Am Enfermagem. 2015; 23: 51-58.

[6] Ke EE, Zhou Q, Wu YL. Emerging paradigms in targeted treatments for Asian patients with NSCLC. Expert Opin Pharmacother. 2015; 16: 1167-1176.

[7] Schiller JH, Harrington D, Belani CP, et al. Comparison of four chemotherapy regimens for advanced non-small-cell lung cancer. N Engl J Med. 2002; 346: 92-98.

[8] Ismael GF, Rosa DD, Mano MS, et al. Novel cytotoxic drugs: old challenges, new solutions. Cancer Treat Rev. 2008; 34: 81-91.

[9] Chabner BA, Roberts TG. Timeline: Chemotherapy and the war on cancer. Nat Rev Cancer. 2005; 5: 65-72.

[10] Domvri K, Zarogoulidis P, Darwiche K, et al. Molecular Targeted Drugs and Biomarkers in NSCLC, the Evolving Role of Individualized Therapy. J Cancer. 2013; 4 (9): 736-754.

[11] Yarden Y, Sliwkowski MX. Untangling the ErbB signalling network. Nat Rev Mol Cell Biol. 2001; 2: 127-137.

[12] Zhang Z, Stiegler AL, Boggon TJ, et al. EGFR-mutated lung cancer: a paradigm of molecular oncology. Oncotarget. 2010; 1: 497-514.

[13] Hanahan D, Weinberg RA. Hallmarks of cancer: the next generation. Cell. 2011; 144: 646-674.

[14] Pao W, Chmielecki J. Rational, biologically based treatment of EGFR-mutant non-small-cell lung cancer. Nat Rev Cancer. 2010; 10: 760-774.

[15] Kobayashi S, Boggon TJ, Dayaram T. EGFR mutation and resistance of non-small-cell lung cancer to gefitinib. N Engl J Med. 2005; 352: 786-792.

[16] Balak MN, Gong Y, Riely GJ. Novel D761Y and common secondary T790M mutations in epidermal growth factor receptor-mutant lung adenocarcinomas with acquired resistance to kinase inhibitors. Clin Cancer Res. 2006; 12: 6494-6501

[17] Bean J, Riely GJ, Balak MN. Acquired resistance to epidermal growth factor receptor kinase inhibitors associated with a novel T854A mutation in a patient with EGFR-mutant lung adenocarcinoma. Clin Cancer Res. 2008; 14: $7519-7525$.

[18] Greig SL. Osimertinib: First Global Approval. Drugs. 2016; 76 (2): 263-273.

[19] Sequist LV, Rolfe L, Allen AR. Rociletinib in EGFR-Mutated Non-Small-Cell Lung Cancer. N Engl J Med. 2015; 373: 578-579.

[20] Bean J, Brennan C, Shih JY. MET amplification occurs with or without T790M mutations in EGFR mutant lung tumors with acquired resistance to gefitinib or erlotinib. Proc Natl Acad Sci USA. 2007; 104: 20932-20937.

[21] Kawano O, Sasaki H, Endo K. PIK3CA mutation status in Japanese lung cancer patients. Lung Cancer. 2006; 54: 209-215.

[22] Zhou J, Wang J, Zeng Y. Implication of Epithelial-Mesenchymal Transition in IGF1R-Induced Resistance to EGFR-TKIs in Advanced Non-Small Cell Lung Cancer. Oncotarget. 2015; 6 (42): 44332-44345.

[23] Yazdi MH, Faramarzi MA, Nikfar S, et al. A Comprehensive Review of Clinical Trials on EGFR Inhibitors Such as Cetuximab and Panitumumab as
Monotherapy and in Combination for Treatment of Metastatic Colorectal Cancer. Avicenna J Med Biotechnol. 2015; 7: 134-144.

[24] Saadeddin A. Radiotherapy for NSCLC: review of conventional and new treatment techniques. J Infect Public Health. 2012; 5 (Suppl 1): S45-S49.

[25] Chinnaiyan P, Huang S, Vallabhaneni G, et al. Mechanisms of enhanced radiation response following epidermal growth factor receptor signaling inhibition by erlotinib (Tarceva). Cancer Res. 2005; 65 (8): 3328-3335.

[26] Kim JC, Ali MA, Nandi A, et al. Correlation of HER1/EGFR expression and degree of radiosensitizing effect of the HER1/EGFR-tyrosine kinase inhibitor erlotinib. Indian J Biochem Biophys. 2005; 42 (6): 358-365.

[27] Baumann M, Krause M, Dikomey E, et al. EGFR-targeted anti-cancer drugs in radiotherapy: preclinical evaluation of mechanisms. Radiother Oncol. 2007; 83 (3): 238-248

[28] Bhardwaj B, Revannasiddaiah S, Bhardwaj H, et al. Molecular targeted therapy to improve radiotherapeutic outcomes for non-small cell lung carcinoma. Ann Transl Med. 2016; 4 (3): 50.

[29] Zabel A, Debus J. Treatment of brain metastases from non-small-cell lung cancer (NSCLC): radiotherapy. Lung Cancer. 2004; 45 (Suppl 2): S247-S252.

[30] Greig NH, Soncrant TT, Shetty HU, et al. Brain uptake and anticancer activities of vincristine and vinblastine are restricted by their low cerebrovascular permeability and binding to plasma constituents in rat. Cancer Chemother Pharmacol. 1990; 26 (4): 263-268.

[31] Lassman AB, Rossi MR, Raizer JJ, et al. Molecular study of malignant gliomas treated with epidermal growth factor receptor inhibitors: tissue analysis from North American Brain Tumor Consortium Trials 01-03 and 00-01. Clin Cancer Res. 2005; 11 (21): 7841-7850.

[32] Cai Y, Wang JY, Liu H. Clinical observation of whole brain radiotherapy concomitant with targeted therapy for brain metastasis in non-small cell lung cancer patients with chemotherapy failure. Asian Pac J Cancer Prev. 2013; 14 (10): 5699-5703.

[33] Fan Y, Huang Z, Fang L, et al. A phase II study of icotinib and whole-brain radiotherapy in Chinese patients with brain metastases from non-small cell lung cancer. Cancer Chemother Pharmacol. 2015; 76 (3): 517-523.

[34] Lu Y, Fan Y. Combined action of EGFR tyrosine kinase inhibitors and whole-brain radiotherapy on EGFR-mutated non-small-cell lung cancer patients with brain metastasis. Onco Targets Ther. 2016; 9: 1135-1143.

[35] Sperduto PW, Wang M, Robins HI, et al. A phase 3 trial of whole brain radiation therapy and stereotactic radiosurgery alone versus WBRT and SRS with temozolomide or erlotinib for non-small cell lung cancer and 1 to 3 brain metastases: Radiation Therapy Oncology Group 0320. Int J Radiat Oncol Biol Phys. 2013; 85 (5): 1312-1318.

[36] Li YS, Jiang BY, Yang JJ, et al. Leptomeningeal Metastases in Patients with NSCLC with EGFR Mutations. J Thorac Oncol. 2016; 11 (11): 1962-1969.

[37] Lind JS, Lagerwaard FJ, Smit EF, et al. Phase I study of concurrent whole brain radiotherapy and erlotinib for multiple brain metastases from non-small-cell lung cancer. Int J Radiat Oncol Biol Phys. 2009; 74 (5): 1391-1396.

[38] Tan CS, Cho BC, Soo RA, et al. Treatment options for EGFR mutant NSCLC with CNS involvement-Can patients BLOOM with the use of next generation EGFR TKIs? Lung Cancer. 2017; 108: 29-37.

[39] Mok TS, Wu Y-L, Ahn M-J, et al. Osimertinib or Platinum-Pemetrexed in EGFR T790M-Positive Lung Cancer. N Engl J Med. 2017; 376 (7): 629-640.

[40] [Internet] National Comprehensive Cancer Network. (NCCN) Clinical Practice Guidelines in Oncology. Non-Small Cell Lung Cancer, Version 2. 2016. https://www.nccn.org/professionals/physician_gls/pdf/nscl.pdf. Accessed 24 Nov. 2016.

[41] Lee JG, Wu R. Erlotinib-cisplatin combination inhibits growth and angiogenesis through c-MYC and HIF-1a in EGFR-mutated lung cancer in vitro and in vivo. Neoplasia. 2015; 17 (2): 190-200.

[42] Jänne PA, Wang X, Socinski MA, et al. Randomized phase II trial of erlotinib alone or with carboplatin and paclitaxel in patients who were never or light former smokers with advanced lung adenocarcinoma: CALGB 30406 trial. J Clin Oncol. 2012; 30 (17): 2063-2069.

[43] Tsai CM, Chen JT, Stewart DJ, et al. Antagonism between gefitinib and cisplatin in non-small cell lung cancer cells: why randomized trials failed. J Thorac Oncol. 2011; 6 (3): 559-568

[44] Liu JT, Li WC, Gao S, et al. Autophagy Inhibition Overcomes the Antagonistic Effect Between Gefitinib and Cisplatin in Epidermal Growth Factor Receptor Mutant Non--Small-Cell Lung Cancer Cells. Clin Lung Cancer. 2015; 16 (5): e55-e66.

[45] Luk PP, Galettis P, Links M. ERK phosphorylation predicts synergism between gemcitabine and the epidermal growth factor receptor inhibitor AG1478. Lung Cancer. 2011; 73 (3): 274-282.

[46] Michael M, White SC, Abdi E, et al. Multicenter randomized, open-label phase II trial of sequential erlotinib and gemcitabine compared with gemcitabine monotherapy as first-line therapy in elderly or ECOG PS two patients with advanced NSCLC. Asia Pac J Clin Oncol. 2015; 11 (1): 4-14.

[47] Yan H, Li H, Li Q, et al. The Efficacy of Synchronous Combination of Chemotherapy and EGFR TKIs for the First-Line Treatment of NSCLC: A Systematic Analysis. PLoS One. 2015; 10 (8): e0135829.

[48] Zinner RG, Fossella FV, Herbst RS. Pemetrexed in advanced NSCLC: a review of the clinical data. Oncology (Williston Park). 2004; 18 (Suppl 5): S54-S62.

[49] Wu M, Yuan Y, Pan YY, et al. Combined gefitinib and pemetrexed overcome the acquired resistance to epidermal growth factor receptor tyrosine kinase inhibitors in non-small cell lung cancer. Mol Med Rep. 2014; 10 (2): 931-938. 
[50] La Monica S, Madeddu D, Tiseo M, et al. Combination of Gefitinib and Pemetrexed Prevents the Acquisition of TKI Resistance in NSCLC Cell Lines Carrying EGFR-Activating Mutation. J Thorac Oncol. 2016; 11 (7): 1051-1063.

[51] Yoshimura N, Kudoh S, Mitsuoka S, et al. Phase II study of a combination regimen of gefitinib and pemetrexed as first-line treatment in patients with advanced non-small cell lung cancer harboring a sensitive EGFR mutation. Lung Cancer. 2015; 90 (1): 65-70.

[52] An C, Zhang J, Chu H, et al. Study of Gefitinib and Pemetrexed as First-Line Treatment in Patients with Advanced Non-Small Cell Lung Cancer Harboring EGFR Mutation. Pathol Oncol Res. 2016; 22 (4): 763-768.

[53] Cheng Y, Murakami H, Yang PC, et al. Randomized Phase II Trial of Gefitinib With and Without Pemetrexed as First-Line Therapy in Patients With Advanced Nonsquamous Non-Small-Cell Lung Cancer With Activating Epidermal Growth Factor Receptor Mutations. J Clin Oncol. 2016; 34 (27): 3258-3266.

[54] Minami S, Kijima T, Hamaguchi M, et al. Phase II study of pemetrexed plus intermittent erlotinib combination therapy for pretreated advanced non-squamous non-small cell lung cancer with documentation of epidermal growth factor receptor mutation status. Lung Cancer. 2013; 82 (2): 271-275.

[55] Kim YH, Nishimura T, Ozasa H, et al. Phase II study of pemetrexed and erlotinib in pretreated nonsquamous, non-small-cell lung cancer patients without an EGFR mutation. Chemotherapy. 2013; 59 (6): 414-419.

[56] Kiselyov A, Balakin KV, Tkachenko SE. VEGF/VEGFR signalling as a target for inhibiting angiogenesis. Expert Opin Investig Drugs. 2007; 16: 83-107.

[57] Ouyang L, Chen Y, Wang XY, et al. Polygonatum odoratum lectin induces apoptosis and autophagy via targeting EGFR-mediated Ras-Raf-MEK-ERK pathway in human MCF-7 breast cancer cells. Phytomedicine. 2014; 21: 1658-1665.

[58] Petrulea MS, Plantinga TS, Smit JW, et al. PI3K/Akt/mTOR: A promising therapeutic target for non-medullary thyroid carcinoma. Cancer Treat Rev. 2015; 41: 707-713.

[59] O'Shea JJ, Schwartz DM, Villarino AV, et al. The JAK-STAT pathway: impact on human disease and therapeutic intervention. Annu Rev Med. 2015; 66: 311-328

[60] Cabebe E, Wakelee H. Role of anti-angiogenesis agents in treating NSCLC: focus on bevacizumab and VEGFR tyrosine kinase inhibitors. Curr Treat Options Oncol. 2007; 8 (1): 15-27.

[61] Fontanini G, Vignati S, Boldrini L, et al. Vascular endothelial growth factor is associated with neovascularization and influences progression of non-small cell lung carcinoma. Clin Cancer Res. 1997; 3 (6): 861-865.

[62] Gentzler RD, Yentz SE, Patel JD. Bevacizumab in advanced NSCLC: chemotherapy partners and duration of use. Curr Treat Options Oncol. 2013; 14 (4): 595-609.

[63] Li H, Takayama K, Wang S, et al. Addition of bevacizumab enhances antitumor activity of erlotinib against non-small cell lung cancer xenografts depending on VEGF expression. Cancer Chemother Pharmacol. 2014; 74 (6): 1297-1305.

[64] Sato $T$, Kato $T$, Nishio $M$, et al Erlotinib alone or with bevacizumab as first-line therapy in patients with advanced non-squamous non-small-cell lung cancer harbouring EGFR mutations (JO25567): an open-label, randomised, multicentre, phase 2 study. Lancet Oncol. 2014; 15 (11): 1236-1244.

[65] Ichihara E, Hotta K, Nogami N, et al. Phase II trial of gefitinib in combination with bevacizumab as first-line therapy for advanced non-small cell lung cancer with activating EGFR gene mutations: the Okayama Lung Cancer Study Group Trial 1001. J Thorac Oncol. 2015; 10 (3): 486-491.

[66] Rosell R, Dafni U, Felip E, et al. Erlotinib and bevacizumab in patients with advanced non-small-cell lung cancer and activating EGFR mutations (BELIEF): an international, multicentre, single-arm, phase 2 trial. Lancet Respir Med. 2017; 5 (5): 435-444

[67] Gridelli C, Rossi A, Ciardiello F, et al. BEVERLY: Rationale and Design of a Randomized Open-Label Phase III Trial Comparing Bevacizumab Plus Erlotinib Versus Erlotinib Alone as First-Line Treatment of Patients With EGFR-Mutated Advanced Nonsquamous Non-Small-Cell Lung Cancer. Clin Lung Cancer. 2016; 17 (5): 461-465.

[68] Tanaka S, Sakamori Y, Niimi M, et al. Design paper: a phase II study of bevacizumab and erlotinib in patients with non-squamous non-small cell lung cancer that is refractory or relapsed after 1-2 previous treatment (BEST). Trials. 2011; 12: 120.

[69] Morabito A, Piccirillo MC, Falasconi F, et al. Vandetanib (ZD6474), a dual inhibitor of vascular endothelial growth factor receptor (VEGFR) and epidermal growth factor receptor (EGFR) tyrosine kinases: current status and future directions. Oncologist. 2009; 14 (4): 378-390.

[70] Loriot Y, Mordant P, Dorvault N, et al. BMS-690514, a VEGFR and EGFR tyrosine kinase inhibitor, shows anti-tumoural activity on non-small-cell lung cancer xenografts and induces sequence-dependent synergistic effect with radiation. Br J Cancer. 2010; 103 (3): 347-353.

[71] Soria JC, Baselga J, Hanna N, et al. Phase I-IIa study of BMS-690514, an EGFR, HER-2 and -4 and VEGFR-1 to -3 oral tyrosine kinase inhibitor, in patients with advanced or metastatic solid tumours. Eur J Cancer. 2013; 49 (8): $1815-1824$

[72] Neal JW, Dahlberg SE, Wakelee HA, et al. Erlotinib, cabozantinib, or erlotinib plus cabozantinib as second-line or third-line treatment of patients with EGFR wild-type advanced non-small-cell lung cancer (ECOG-ACRIN 1512): a randomised, controlled, open-label, multicentre, phase 2 trial. Lancet Oncol. 2016; 17 (12): 1661-1671.

[73] Prigent SA, Lemoine NR. The type 1 (EGFR-related) family of growth factor receptors and their ligands. Prog Growth Factor Res. 1992; 4 (1): 1-24.

[74] Normanno N, Maiello MR, De Luca A. Epidermal growth factor receptor tyrosine kinase inhibitors (EGFR-TKIs): simple drugs with a complex mechanism of action. J Cell Physiol. 2003; 194 (1): 13-19.

[75] Sartore-Bianchi A, Cerea G, Schiavetto I, et al. Anti-EGFR monoclonal antibodies in the treatment of non-small cell lung cancer. Ann Oncol. 2006; 17 (Suppl 2): S49-S51.

[76] Cavazzoni A, Alfieri RR, Cretella D, et al. Combined use of anti-ErbB monoclonal antibodies and erlotinib enhances antibody-dependent cellular cytotoxicity of wild-type erlotinib-sensitive NSCLC cell lines. Mol Cancer. 2012; 11: 91.

[77] Wang M, Zhao J, Zhang LM, et al. Combined Erlotinib and Cetuximab overcome the acquired resistance to epidermal growth factor receptors tyrosine kinase inhibitor in non-small-cell lung cancer. J Cancer Res Clin Oncol. 2012; 138 (12): 2069-2077.

[78] Ramalingam S, Forster J, Naret C, et al. Dual inhibition of the epidermal growth factor receptor with cetuximab, an IgG1 monoclonal antibody, and gefitinib, a tyrosine kinase inhibitor, in patients with refractory non-small cell lung cancer (NSCLC): a phase I study. J Thorac Oncol. 2008; 3 (3): 258-264.

[79] Kim SH, Shim HS, Cho J, et al. A phase I trial of gefitinib and nimotuzumab in patients with advanced non-small cell lung cancer (NSCLC). Lung Cancer. 2013; 79 (3): 270-275

[80] Lin CH, Lin MT, Kuo YW, et al. Afatinib combined with cetuximab for lung adenocarcinoma with leptomeningeal carcinomatosis. Lung Cancer. 2014; 85 (3): $479-480$

[81] Ribeiro Gomes J, Cruz MR. Combination of afatinib with cetuximab in patients with EGFR-mutant non-small-cell lung cancer resistant to EGFR inhibitors. Onco Targets Ther. 2015; 8: 1137-1142.

[82] Neal JW, Heist RS, Fidias P. Cetuximab monotherapy in patients with advanced non-small cell lung cancer after prior epidermal growth factor receptor tyrosine kinase inhibitor therapy. J Thorac Oncol. 2010; 5 (11): $1855-1858$

[83] Mar N, Vredenburgh JJ, Wasser JS. Targeting HER2 in the treatment of non-small cell lung cancer. Lung Cancer. 2015; 87 (3): 220-225.

[84] Hirata A, Hosoi F, Miyagawa M, et al. HER2 overexpression increases sensitivity to gefitinib, an epidermal growth factor receptor tyrosine kinase inhibitor, through inhibition of HER2/HER3 heterodimer formation in lung cancer cells. Cancer Res. 2005; 65 (10): 4253-4260.

[85] Nakamura H, Takamori S, Fujii T, et al. Cooperative cell-growth inhibition by combination treatment with ZD1839 (Iressa) and trastuzumab (Herceptin) in non-small-cell lung cancer. Cancer Lett. 2005; 230 (1): 33-46.

[86] Friess T, Scheuer W, Hasmann M. Combination treatment with erlotinib and pertuzumab against human tumor xenografts is superior to monotherapy. Clin Cancer Res. 2005; 11 (14): 5300-5309.

[87] Hughes B, Mileshkin L, Townley P, et al. Pertuzumab and erlotinib in patients with relapsed non-small cell lung cancer: a phase II study using 18F-fluorodeoxyglucose positron emission tomography/computed tomography imaging. Oncologist. 2014; 19 (2): 175-176.

[88] Ricciardi GR, Russo A, Franchina T, et al. NSCLC and HER2: between lights and shadows. J Thorac Oncol. 2014; 9 (12): 1750-1762.

[89] Pao W, Wang TY, Riely GJ, et al. KRAS mutations and primary resistance of lung adenocarcinomas to gefitinib or erlotinib. PLoS Med. 2015; 2 (1): e17.

[90] Paik PK, Arcila ME, Fara M, et al. Clinical characteristics of patients with lung adenocarcinomas harboring BRAF mutations. J Clin Oncol. 2011; 29 (15): 2046-2051.

[91] Martinelli E, Troiani T, Morgillo F, et al. Synergistic antitumor activity of sorafenib in combination with epidermal growth factor receptor inhibitors in colorectal and lung cancer cells. Clin Cancer Res. 2010; 16 (20): 4990-5001.

[92] Balko JM, Jones BR, Coakley VL, et al. MEK and EGFR inhibition demonstrate synergistic activity in EGFR-dependent NSCLC. Cancer Biol Ther. 2009; 8 (6): 522-530

[93] Song JY, Kim CS, Lee JH, et al. Dual inhibition of MEK1/2 and EGFR synergistically induces caspase-3-dependent apoptosis in EGFR inhibitor-resistant lung cancer cells via BIM upregulation. Invest New Drugs. 2013; 31 (6): 1458-1465.

[94] Li S, Chen S, Jiang Y, et al. Synergistic interaction between MEK inhibitor and gefitinib in EGFR-TKI-resistant human lung cancer cells. Oncol Lett. 2015; 10 (4): 2652-2656

[95] Garnock-Jones KP. Cobimetinib: First Global Approval. Drugs. 2015; 75 (15): 1823-1830.

[96] Garon EB, Finn RS, Hosmer W, et al. Identification of common predictive markers of in vitro response to the Mek inhibitor selumetinib (AZD6244; ARRY-142886) in human breast cancer and non-small cell lung cancer cell lines. Mol Cancer Ther. 2010; 9 (7): 1985-1994

[97] [Internet] US National Library of Medicine. ClinicalTrials.gov [online]. https://clinicaltrials.gov/ct2/show/NCT01229150.

[98] [Internet] US National Library of Medicine. ClinicalTrials.gov [online]. https://clinicaltrials.gov/ct2/show/NCT01859026.

[99] Carter CA, Rajan A, Keen C, et al. Selumetinib with and without erlotinib in KRAS mutant and KRAS wild-type advanced nonsmall-cell lung cancer. Ann Oncol. 2016; 27 (4): 693-699. 
[100]Das M. Selumetinib does not improve survival outcomes in NSCLC. Lancet Oncol. 2017; 18 (6): e313.

[101]Luo J, Manning BD, Cantley LC. Targeting the PI3K-Akt pathway in human cancer: rationale and promise. Cancer Cell. 2003; 4: 257-262.

[102]Pérez-Ramírez C, Cañadas-Garre M, Molina MÁ, et al. PTEN and PI3K/AKT in non-small-cell lung cancer. Pharmacogenomics. 2015; 16 (16): 1843-1862.

[103]Kokubo Y, Gemma A, Noro R, et al. Reduction of PTEN protein and loss of epidermal growth factor receptor gene mutation in lung cancer with natural resistance to gefitinib (IRESSA). Br J Cancer. 2005; 92 (9): 1711-1719.

[104]Karachaliou N, Codony-Servat J, Teixidó C, et al. BIM and mTOR expression levels predict outcome to erlotinib in EGFR-mutant non-small-cell lung cancer. Sci Rep. 2015; 5: 17499.

[105]Wang YC, Kulp SK, Wang D, et al. Targeting endoplasmic reticulum stress and Akt with OSU-03012 and gefitinib or erlotinib to overcome resistance to epidermal growth factor receptor inhibitors. Cancer Res. 2008; 68 (8): 2820-2830.

[106]Dong S, Zhang XC, Cheng H, et al. Everolimus synergizes with gefitinib in non-small-cell lung cancer cell lines resistant to epidermal growth factor receptor tyrosine kinase inhibitors. Cancer Chemother Pharmacol. 2012; 70 (5): 707-716.

[107][Internet] US National Library of Medicine. ClinicalTrials.gov [online]. https://clinicaltrials.gov/ct2/show/NCT01570296.

[108][Internet] US National Library of Medicine. ClinicalTrials.gov [online]. https://clinicaltrials.gov/ct2/show/NCT00777699.

[109]Jr. Lara PN, Longmate J, Mack PC, et al. Phase II Study of the AKT Inhibitor MK-2206 plus Erlotinib in Patients with Advanced Non-Small Cell Lung Cancer Who Previously Progressed on Erlotinib. Clin Cancer Res. 2015; 21 (19): 4321-4326.

[110]Forcello N, Saraiya N. Idelalisib: The First-in-Class Phosphatidylinositol 3-Kinase Inhibitor for Relapsed CLL, SLL, and Indolent NHL. J Adv Pract Oncol. 2014; 5: 455-459.

[111]Hasskarl J. Everolimus. Recent Results Cancer Res. 2014; 201: 373-392.

[112]Schulze M, Stock C, Zaccagnini M, et al. Temsirolimus. Recent Results Cancer Res. 2014; 201: 393-403.

[113]Jacot JL, Sherris D. Potential Therapeutic Roles for Inhibition of the PI3K/Akt/mTOR Pathway in the Pathophysiology of Diabetic Retinopathy. J Ophthalmol. 2011; 2011: 589813.

[114]Garajová I, Giovannetti E, Biasco G, et al. c-Met as a Target for Personalized Therapy. Transl Oncogenomics. 2015; 7 (Suppl 1): S13-S31.

[115]Puri N, Salgia R. Synergism of EGFR and c-Met pathways, cross-talk and inhibition, in non-small cell lung cancer. J Carcinog. 2008; 7: 9 .

[116]Zhang YW, Staal B, Essenburg C, et al. Strengthening context-dependent anticancer effects on non-small cell lung carcinoma by inhibition of both MET and EGFR. Mol Cancer Ther. 2013; 12 (8): 1429-1441.

[117]Stabile LP, Rothstein ME, Keohavong P, et al. Targeting of Both the c-Met and EGFR Pathways Results in Additive Inhibition of Lung Tumorigenesis in Transgenic Mice. Cancers (Basel). 2010; 2 (4): 2153-2170.

[118]Lara MS, Holland WS, Chinn D, et al. Preclinical Evaluation of MET Inhibitor INC-280 With or Without the Epidermal Growth Factor Receptor Inhibitor Erlotinib in Non-Small-Cell Lung Cancer. Clin Lung Cancer. 2017; 18 (3): 281-285.

[119]Friese-Hamim M, Bladt F, Locatelli G, et al. The selective c-Met inhibitor tepotinib can overcome epidermal growth factor receptor inhibitor resistance mediated by aberrant c-Met activation in NSCLC models. Am J Cancer Res. 2017; 7 (4): 962-972.

[120]Scagliotti GV, Novello S, Schiller JH, et al. Rationale and design of MARQUEE: a phase III, randomized, double-blind study of tivantinib plus erlotinib versus placebo plus erlotinib in previously treated patients with locally advanced or metastatic, nonsquamous, non-small-cell lung cancer. Clin Lung Cancer. 2012; 13 (5): 391-395.

[121]Spigel DR, Ervin TJ, Ramlau RA, et al. Randomized phase II trial of Onartuzumab in combination with erlotinib in patients with advanced non-small-cell lung cancer. J Clin Oncol. 2013; 31 (32): 4105-4114.

[122]Tarhini AA, Rafique I, Floros T, et al. Phase $1 / 2$ study of rilotumumab (AMG 102), a hepatocyte growth factor inhibitor, and erlotinib in patients with advanced non-small cell lung cancer. Cancer. 2017; 123 (15): 2936-2944.

[123]Sequist LV, von Pawel J, Garmey EG, et al. Randomized phase II study of erlotinib plus tivantinib versus erlotinib plus placebo in previously treated non-small-cell lung cancer. J Clin Oncol. 2011; 29 (24): 3307-3315.

[124]Scagliotti G, von Pawel J, Novello S, et al. Phase III Multinational, Randomized, Double-Blind, Placebo-Controlled Study of Tivantinib (ARQ 197) Plus Erlotinib Versus Erlotinib Alone in Previously Treated Patients With Locally Advanced or Metastatic Nonsquamous Non-Small-Cell Lung Cancer. J Clin Oncol. 2015; 33 (24): 2667-2674.

[125] Ou SI, Govindan R, Eaton KD, et al. Phase I Results from a Study of Crizotinib in Combination with Erlotinib in Patients with Advanced Nonsquamous Non-Small Cell Lung Cancer. J Thorac Oncol. 2017; 12 (1): 145-151.

[126]Yoshioka H, Azuma K, Yamamoto N, et al. A randomized, double-blind, placebo-controlled, phase III trial of erlotinib with or without a c-Met inhibitor tivantinib (ARQ 197) in Asian patients with previously treated stage IIIB/IV nonsquamous nonsmall-cell lung cancer harboring wild-type epidermal growth factor receptor (ATTENTION study). Ann Oncol. 2015; 26 (10):2066-2072.

[127]Azuma K, Hirashima T, Yamamoto N, et al. Phase II study of erlotinib plus tivantinib (ARQ 197) in patients with locally advanced or metastatic EGFR mutation-positive non-small-cell lung cancer just after progression on EGFR-TKI, gefitinib or erlotinib. ESMO Open. 2016; 1 (4): e000063.

[128]Sano Y, Hashimoto E, Nakatani N, et al. Combining onartuzumab with erlotinib inhibits growth of non-small cell lung cancer with activating EGFR mutations and HGF overexpression. Mol Cancer Ther. 2015; 14 (2): 533-541.

[129] Ou SH, Agarwal N, Ali SM. High MET amplification level as a resistance mechanism to osimertinib (AZD9291) in a patient that symptomatically responded to crizotinib treatment post-osimertinib progression. Lung Cancer. 2016; 98: 59-61.

[130]Passiglia F, Bronte G, Castiglia M, et al. Prognostic and predictive biomarkers for targeted therapy in NSCLC: for whom the bell tolls. Expert Opin Biol Ther. 2015; 15 (11): 1553-1566.

[131]Bhattacharya B, Mohd Omar MF, Soong R. The Warburg effect and drug resistance. Br J Pharmacol. 2016; 173 (6): 970-979.

[132]Langhammer S, Scheerer J. Breaking the crosstalk of the cellular tumorigenic network: Hypothesis for addressing resistances to targeted therapies in advanced NSCLC. Oncotarget. 2017; 8 (26): 43555-43570.

[133] Stambrook PJ, Maher J, Farzaneh F. Cancer Immunotherapy: Whence and Whither. Mol Cancer Res. 2017; 15 (6): 635-650.

[134]Zhou TC, Sankin AI, Porcelli SA, et al. A review of the PD-1/PD-L1 checkpoint in bladder cancer: From mediator of immune escape to target for treatment. Urol Oncol. 2017; 35 (1): 14-20.

[135]Naylor EC, Desani JK, Chung PK. Targeted Therapy and Immunotherapy for Lung Cancer. Surg Oncol Clin N Am. 2016; 25 (3): 601-609.

[136]Malhotra J, Jabbour SK, Aisner J. Current state of immunotherapy for non-small cell lung cancer. Transl Lung Cancer Res. 2017; 6 (2): 196-211.

[137]Saito M, Suzuki H, Kono K, et al. Treatment of lung adenocarcinoma by molecular-targeted therapy and immunotherapy. Surg Today 2017; [Epub ahead of print].

[138]Colli LM, Machiela MJ, Zhang H, et al. Landscape of Combination Immunotherapy and Targeted Therapy to Improve Cancer Management. Cancer Res. 2017; 77 (13): 3666-3671.

[139]Su Q, Sun Z, Zhang C, et al. PD-1/PD-L1 antibodies efficacy and safety versus docetaxel monotherapy in advanced NSCLC patients, after first-line treatment option: systems assessment. Oncotarget 2017; [Epub ahead of print]

[140]Chen N, Fang W, Zhan J, et al. Upregulation of PD-L1 by EGFR Activation Mediates the Immune Escape in EGFR-Driven NSCLC: Implication for Optional Immune Targeted Therapy for NSCLC Patients with EGFR Mutation. J Thorac Oncol. 2015; 10 (6): 910-923.

[141]D'Incecco A, Andreozzi M, Ludovini V, et al. PD-1 and PD-L1 expression in molecularly selected non-small-cell lung cancer patients. Br J Cancer. 2015; 112 (1): 95-102.

[142]Ahn MJ, Sun JM, Lee SH, et al. EGFR TKI combination with immunotherapy in non-small cell lung cancer. Expert Opin Drug Saf. 2017; 16 (4): 465-469.

[143] Herbst RS, Baas P, Kim DW, et al. Pembrolizumab versus docetaxel for previously treated, PD-L1-positive, advanced non-small-cell lung cancer (KEYNOTE-010): a randomised controlled trial. Lancet. 2016; 387 (10027): $1540-1550$

[144]Borghaei H, Paz-Ares L, Horn L, et al. Nivolumab versus Docetaxel in Advanced Nonsquamous Non-Small-Cell Lung Cancer. N Engl J Med. 2015; 373 (17): 1627-1639.

[145]Salvi S, Fontana V, Boccardo S, et al. Evaluation of CTLA-4 expression and relevance as a novel prognostic factor in patients with non-small cell lung cancer. Cancer Immunol Immunother. 2012; 61 (9): 1463-1472.

[146] Horinouchi H, Yamamoto N, Fujiwara Y, et al. Phase I study of ipilimumab in phased combination with paclitaxel and carboplatin in Japanese patients with non-small-cell lung cancer. Invest New Drugs. 2015; 33 (4): 881-889.

[147]Zatloukal P, Heo DS, Park K, et al. Randomized phase II clinical trial comparing tremelimumab $(\mathrm{CP}-675,206)$ with best supportive care (BSC) following first-line platinum-based therapy in patients (pts) with advanced non-small cell lung cancer (NSCLC). J Clin Oncol (Meeting Abstracts). 2009; 27 (Suppl 15): S8071.

[148]Kim ES. Olmutinib: First Global Approval. Drugs. 2016; 76 (11): 1153-1157.

[149]Planchard D, Loriot Y, André F, et al. EGFR-independent mechanisms of acquired resistance to AZD9291 in EGFR T790M-positive NSCLC patients. Ann Oncol. 2015; 26: 2073-2078.

[150]Mérida I, Avila-Flores A. Tumor metabolism: new opportunities for cancer therapy. Clin Transl Oncol. 2016; 8 (10): 711-716.

[151]Fennell DA, Summers Y, Cadranel J, et al. Cisplatin in the modern era: The backbone of first-line chemotherapy for non-small cell lung cancer. Cancer Treat Rev. 2016; 44: 42-50.

[152]Elf SE, Chen J. Targeting glucose metabolism in patients with cancer. Cancer. 2014; 120: 774-780

[153]Medina RA, Owen GI. Glucose transporters: expression, regulation and cancer. Biol Res. 2002; 35: 9-26.

[154]Wu J, Hu L, Wu F, et al. Poor prognosis of hexokinase 2 overexpression in solid tumors of digestive system: a meta-analysis. Oncotarget. 2017; 8 (19): 32332-32344.

[155]Wang P, Sun C, Zhu T, et al. Structural insight into mechanisms for dynamic regulation of PKM2. Protein Cell. 2015; 6: 275-287.

[156]Jha MK, Suk K. Pyruvate dehydrogenase kinase as a potential therapeutic target for malignant gliomas. Brain Tumor Res Treat. 2013; 1: 57-63.

[157]Valvona CJ, Fillmore HL, Nunn PB, et al. The Regulation and Function of Lactate Dehydrogenase A: Therapeutic Potential in Brain Tumor. Brain Pathol. 2016; 26 (1): 3-17. 
[158]Sheng H, Tang W. Glycolysis Inhibitors for Anticancer Therapy: A Review of Recent Patents. Recent Pat Anticancer Drug Discov. 2016; 11 (3): 297-308.

[159]Yang Z, Tam KY. Anti-cancer synergy of dichloroacetate and EGFR tyrosine kinase inhibitors in NSCLC cell lines. Eur J Pharmacol. 2016; 789: 458-467.

[160]Yang Z, Hu X, Zhang S, et al. Pharmacological synergism of 2,2-dichloroacetophenone and EGFR-TKi to overcome TKi-induced resistance in NSCLC cells. Eur J Pharmacol 2017; 815: 80-87. 